\title{
A Study and Construction of Multi-level Fuzzy Comprehensive Evaluation System of Course
}

\author{
L. Tan, T.Q. Jiang, J.P. Hu, J.J. Lian \\ School of Computer \& Information Engineering Beijing \\ Technology and Business University \\ Beijing, China
}

\begin{abstract}
At present, the course quality evaluation method is unitary with a strong subjective character. What is more, the factors are incomprehensive in the evaluation index system. In order to solve the problems, this paper is involved in establishment of a comparatively complete quality evaluation system of course in accordance with the features of present course teaching in colleges and universities on the basis of which invested a method of fuzzy comprehensive evaluation based on Rough Set, that is, on the whole, objective in determination of index factors. On the basis of characteristics of index system structure, generally adopted Rough Set and analytic hierarchy process to calculate level three as well as level one and two index weights, finally achieved the evaluation results through multilevel fuzzy comprehensive evaluation method. Utilized the results that had been obtained through comparison of experiment, the multilevel fuzzy comprehensive evaluation system constructed in this paper is capable to perfectly evaluate teaching effect.
\end{abstract}

Keywords-quality evaluation of course; evaluation index of course quality; Rough Set, fuzzy comprehensive evaluation method; analytic hierarchy process

\section{INTRODUCTION}

Nowadays, there are a quite number of methods for evaluation of teaching quality, such as fuzzy mathematics applied broadly in course quality evaluation [1]. By so doing, the fuzzy mathematics theory is introduced into course evaluation to process data, determine evaluation factors and levels, apply the fuzzy multi-value evaluation method in carrying out course quality evaluation from different angles. By this method, the accuracy of evaluation result of teachers' teaching quality is guaranteed while the Rough Set theory is also well used in teaching quality evaluation [2]. Wang et al [3] through analyzing the experimental teaching characteristics of applicable economic management undergraduate course, by application of Rough Set theory and information entropy concept as well as other related analysis, realized the relative teaching quality comprehensive evaluation model after screening over the initially selected evaluation index, and bestow on normalized index. Whereas Yang et al [4] have explored analytic model of teaching evaluation, upon analysis have respectively established the mathematical model of teaching quality evaluation system with Neural Network theory, fuzzy comprehensive evaluation model and fuzzy comprehensive evaluation model based on modified AHP. Upon comparison of these three related evaluation models, the teaching quality evaluation model has been constructed based on statistics.

\author{
L.L. Shang \\ The second Technology development department China \\ Distance Education Holdings Ltd., CDEL \\ Beijing, China
}

In spite of obvious progress of study on the quality evaluation theory of course teaching [5-6] has been made, due to the varieties of courses and divergence of students, the quality evaluation of course teaching system is not yet maturely formed. Particularly, in theory exploration, rules and system formulation of quality evaluation of course teaching, teaching inspection method and means renew etc., are still under the experimental period that is remained to be perfected.

\section{CONSTRUCTION OF MULTI-LEVEL FUZZY EVALUATION INDEX SYSTEM OF COURSE QUALITY}

\section{A. Index Factors System of Course Quality Evaluation}

Upon careful study and analysis on characteristics of present courses in colleges and universities, it is taken that evaluation index should not just consist of the course checking index only, but also should include the index of teachers' teaching, educational facilities and teacher's conditions etc.. In this paper therefore, all sided information concerning courses has been taken into consideration, finally formulated the following course evaluation index system.

The values of various index factors are taken as follows:

U111-Lecturer, associate professor and professor are graded as 1, 2, 3 respectively.

U121—Number of years' of teaching.

U131-Undergraduate, post graduate, Ph.D. doctorate and post doctorate are graded as 1, 2, 3, 4 respectively.

U141 U145_- Take actual value of each index.
U151 U152_ — Take actual value of each index.

U211 U212_Assess the grades according to completeness of specific index and teachers employment (grades are classified as 1, 2 and 3respectively). Grade is to be fixed as concrete value.

U221 U223_Assess the grades according to completeness of specific index and teachers employment (grade are classified as 1, 2 and 3 respectively). Grade is to be fixed as concrete value.

U311 U317__ Take the students' evaluation result of courses to corresponding teachers.

U411_ Take the average achievement of students.

U421 U423_— Take the course evaluation result. 


\section{B. Fuzzy Comprehensive Evaluation method Based on Rough Set}

1) Factor weight: Weight of Level 1 and Level 2 index factors are calculated with the analytic hierarchy process, the experts provide the judgment matrix of related factors in the rule hierarchy out of the hierarchical structure diagrams, to decide if identity requirement is met, then normalizing treatment is conducted, while Level 3 index factor weight is distributed by rough set approach.

As for weight classification of Level 3 index factors with Rough Set Theory, first of all, to derive specific data of index factor from databank, then cluster that with SOM network so as to get decision attribute based on which to discrete the continuous data, finally carry out calculation of the discrete data with the Rough Set Theory, consequently achieve the factor weight.

The calculation process of factor weight distribution with the rough set in the above flow chart is as the following:

$$
\begin{aligned}
& \text { Object set } U=\left\{u_{1}, u_{2} \cdots, u_{l}\right\} ; \\
& \text { Condition attribute } C=\left\{c_{1}, c_{2} \cdots, c_{n}\right\} \\
& \text { Decisions attribute } D=\left\{d_{1}, d_{2}, d_{3}, d_{4}\right\} .
\end{aligned}
$$

a) Delete condition attribute $c_{i}(i=1,2, \cdots, n)$, and make sure the classification set $Y_{l}$ is the best after condition attribute $c_{i}$ is deleted.

b) Find solution for positive region of various equal value set of decision attributes:

$$
\operatorname{POS}_{C-\left\{c_{i}\right\}}(D)=\left\{C-\left\{c_{i}\right\}\right\} \cdot(D)=\left\{\left\{C-\left\{C_{i}\right\}\right\} \cdot Y_{l}\right\}
$$

c) Calculate the dependent degree of the two attribute sets:

$$
\gamma\left(C-\left\{c_{i}\right\}, D\right)=\left|\operatorname{POS}_{C-\left\{c_{i}\right\}}(D)\right| /|U|
$$

d) Find solution of importance degree of attribute ${ }^{C_{i}}$ :

$$
S G F\left(c_{i}, C, D\right)=\gamma(C, D, \beta)-\gamma\left(C-c_{i}, D, \beta\right)
$$

Calculate weight of each condition attribute:

$$
w_{i}=S G F\left(c_{i}, C, D\right) / \sum_{k=1}^{n} S G F\left(c_{k}, C, D\right)
$$

2) Evaluation method: To evaluate the course teaching quality with fuzzy comprehensive evaluation method, in which the membership function is worked out based on questionnaire survey and characteristics of particular issues. In the respect of selection of fuzzy arithmetic operators, a special consideration of reality features problem is taken into account. In order to avoid information lost, and to comprehensively handle factor weight and the relation matrix, the weighted mean model has been defined. The evaluation grades have been classified as excellent, fairly good, common and poor.

\section{COMPARISON OF EXPERIMENT}

The data obtaining way of teachers' teaching quality of courses.

\section{1) Teachers' data}

The data including teachers' records of formal schooling, academic titles and their papers that have been published etc. collected by the Dean's Office of colleges and universities.

\section{2) Students' evaluation}

The students would have to give their evaluation on their class teachers' teaching quality of courses each term. This evaluation is normally arranged before the terminal examination.

The judgment matrix shall be provided by experts.

Take the data of the following ten courses of discrete mathematics, English, neural network, stochastic process, computer network, university literature, higher mathematics, university physics, linear algebra, $C$ language program design etc., for experiment. Take data of few teachers and courses as the sample data set to calculate each index weight first, to get three level index weight with Rough Set Method, then, calculate the level 1 and 2 index weight with the analytic hierarchy process. Carry out evaluation on six courses with multi-level fuzzy evaluation method. The results are listed as follows:

Discrete mathematics $=\{0.3243,0.3927,0.2684,0.0146\} ;$

English $=\{0.3788,0.1999,0.3341,0.0873\} ;$

Neural network $=\{0.6655,0.0300,0.2610,0.0435\}$;

Stochastic process $=\{0.0503,0.4261,0.3595,0.3595\}$;

Computer network $=\{0.5264,0.0191,0.2431,0.2114\}$;

University literature $=\{0.0621,0.2599,0.5005,0.1775\}$;

Higher mathematics $=\{0.3224,0.1233,0.4138,0.1405\}$;

University physics $=\{0.5008,0.1269,0.1366,0.2357\}$;

Linear algebra $=\{0.5381,0.2027,0.1138,0.1454\}$;

$\mathrm{C}$ language program design $=\{0.0527,0.0375,0.3687$, $0.5412\}$.

The curves comparison of evaluation result by model in this paper and the expert evaluation as shown in the following.
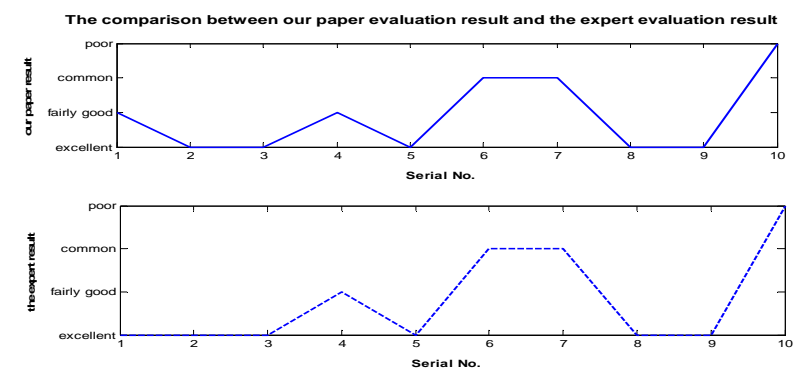

FIGURE I. COMPARISON BETWEEN THE PAPER EVALUATION RESULT AND THE EXPERT EVALUATION RESULT. 
It is clearly seen from the chart and curve comparison, evaluation result by model and that of the experts maintain largely unanimous and the accuracy reached $90 \%$ from where it reflects the reliability and accuracy of model and the orientation of this paper, and it is worthy to further study and application.

\section{CONCLUSION AND PERSPECTIVE}

A comprehensive consideration of both objective and subjective index concerning course teaching is taken in this paper, established an adequately complete evaluation index system of course, and formulated a fuzzy evaluation model in which a multi-level comprehensive evaluation method based on Rough Set that is applied to calculate three-leveled index weight whilst the index weight of Level 1 and level 2 are calculated with the analytic hierarchy process, out of which judgment matrix is provided by experts, and the membership function is constructed by experts based on their experiences and specific issues features. On the basis of the above discussions, by application of multi-level fuzzy comprehensive evaluation method to evaluate courses, whose results are compared with the expert evaluation, further verified the accuracy of this paper model.

\section{ACKNOWLEDGEMENT}

We are grateful for the supported by Beijing Natural Science Foundation (4132025) and Beijing Higher Education Young Elite Teacher Project (YETP1448).

\section{REFERENCES}

[1] H. Zhu, A study[D] on teaching quality evaluation system of colleges and universities, Dalian Science and Engineering University, 2004.

[2] L.Y. Wang, Application of Rough Set in teaching evaluation system, Journal of Hubei Science and Engineering College, 02, pp: 47-50,2014.

[3] Y. Wang, Evaluation method[J] of applied economic undergraduate experimental teaching quality based on Rough Set Theory, Xinyu College Journal, 03, pp: 110-112, 2014.

[4] D.Q. Yang, A study[D] on teaching quality evaluation model and case analysis and application, Hubei, Central China Normal University, 2008.

[5] I X.L. Ye, X.Y. Liu \& S.Y. Xiao, ntegral course checking and evaluation method[J] based on fuzzy mathematics, Occupation Journal, 24, 2013.

[6] M. Li, A study on internal teaching quality monitoring system for China's research model university, China Aviation and Space Science University Journal (social science Column) , 27 (1), pp:105-120, 2014. 